GENETICS

\title{
Evidence of altered RNA stirs debate
}

\section{Sceptics question find that upends biology's 'central dogma'.}

\section{BY ERIKA CHECK HAYDEN}

A funny thing happened on the way to the ribosome. That's the essence of a controversial paper concluding that messenger RNA - the molecular middleman that carries information from a cell's DNA to its protein-making machinery - is routinely and systematically altered by unknown mechanisms before its genetic instructions can be read. The paper, published in Science last week (M. Li et al. Science doi:10.1126/science.1207018;2011), is already drawing pointed reviews from computational biologists, who cite possible flaws that could undermine the authors' claims.

If verified, the findings would require a rewrite of the 'central dogma' of molecular biology, which posits that the RNA transcripts that carry genetic information to the ribosome, where they are used as templates for protein assembly, are generally faithful matches to the original DNA. A revised version of the picture would include an 'RNA editing' step along the way, which replaces individual letters in the genetic code and changes the resulting proteins (see 'Unmatched messages'). Such a step would allow cells to generate much more diversity from the

standard DNA tool kit than previously thought. Vivian Cheung of the University of Pennsylvania in Philadelphia led the work, which involved examining the RNA transcripts and DNA sequences of 27 people who were sequenced in the 1000 Genomes Project and the International HapMap Project. The team found more than 10,000 sites in exons - regions of messenger RNA that have been transcribed from DNA - in which the DNA and RNA sequences

"The big

challenge now is to sort out the molecular mechanism." did not match. The same mismatches occurred in different people, suggesting that they were not random mistakes in transcrip-

tion. Cheung's team also found proteins made from the 'mismatched' RNAs.

"Once we saw that these differences were translated into protein sequences, we were pretty certain that they were biologically derived," Cheung says.

RNA editing - a process that changes the identity of an RNA base after it has been transcribed from a DNA sequence - is not a new discovery. An enzyme called ADAR, for instance, induces mismatches in human cells

\section{UNMATCHED MESSAGES}

The swapping out of a single base in a section of messenger RNA alters the genetic information transcribed from the DNA before it is translated into a protein.

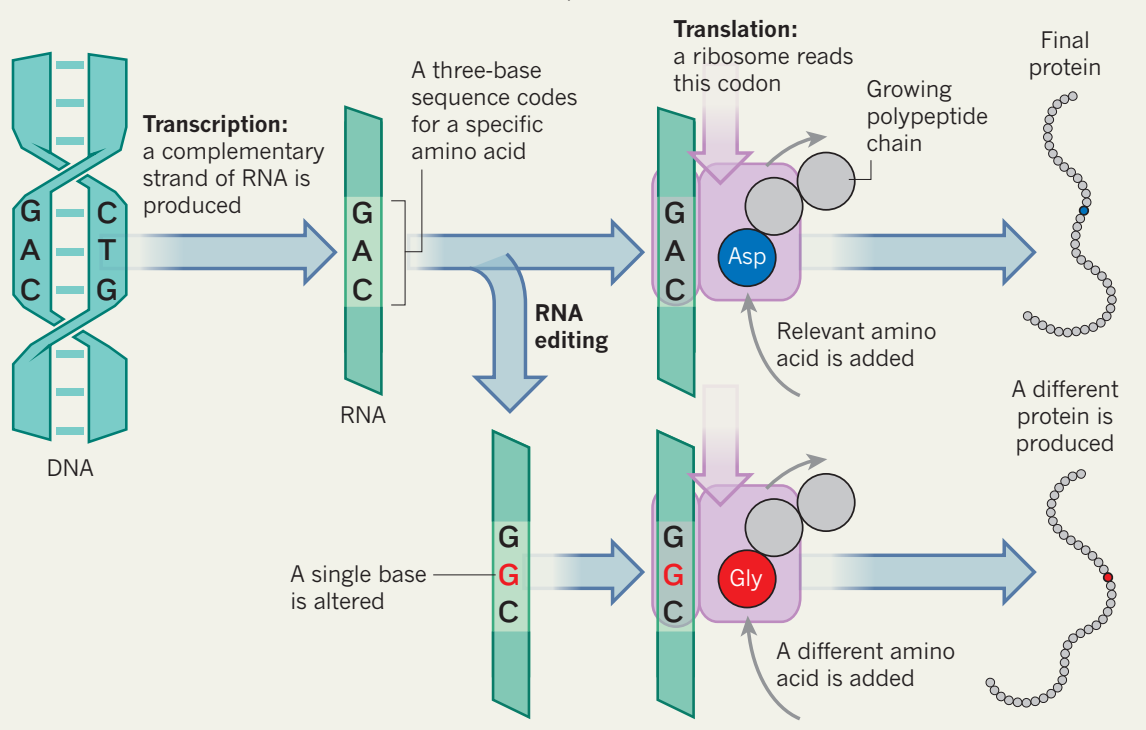

by replacing the base adenosine with another molecule that is then read as guanine when the RNA is used to code for a protein. RNA editing also occurs in plants and human parasites.

But the extent of RNA editing posited by the Science paper is extraordinary; its authors estimate that each person has about 1,065 mismatches - sites the authors call "RNA-DNA differences”, or RDDs. Some of the mismatches involve base changes that are not produced by known RNA-editing mechanisms, suggesting that undiscovered mechanisms are at work.

"This suggests a completely different layer of gene regulation at the RNA level," says molecular biologist Kazuko Nishikura at the Wistar Institute in Philadelphia. "The big challenge now is to sort out the molecular mechanism for how these RNA sequence alterations can be achieved."

Others remain sceptical. Comparative genomicist Lior Pachter at the University of California, Berkeley, has studied how the highthroughput sequencing machines that Cheung's team used to sequence RNA make systematic errors when sequencing DNA and RNA. He says that some of Cheung's mismatches occur at sites that are prone to systematic RNA sequencing errors, but others do not.

And in a post on the blog 'genomes unzipped' on 20 May, Joe Pickrell, a graduate student working with human geneticist Jonathan Pritchard at the University of Chicago, Illinois, described another potential source of error. Pickrell said that multiple regions of similar DNA in the human genome can make it difficult to trace the origin of a short stretch of RNA to a specific DNA sequence, creating the illusion of DNA-RNA differences. "If the authors are accidentally attributing RNA from two different regions of the genome to the same DNA region, they could falsely infer RNA editing," Pickrell said. "I think many of their results could be the result of errors in identifying the correct genomic origin of their sequencing reads."

Other researchers are combing through their own data and waiting to see the results of follow-up work that will determine whether the concerns raised by Pachter, Pickrell and others are valid. Meanwhile, Chueng says, "we are glad to see that our colleagues are already using our data”.

If confirmed, Cheung's work has important implications for biology and for the way that researchers study genomics. Chris Gunter, director of research affairs at the HudsonAlpha Institute for Biotechnology in Huntsville, Alabama, says that RNA editing might have implications for the genetic origins of disease, if it turns out that the control of how

$\rightarrow$ NATURE.COM For more on the complexity of the genome, see: go.nature.com/unyfgr much editing occurs is inherited.

"This could make our jobs as geneticists more problematic and more interesting," she says. 